\title{
Optimization of the GSFC TROPOZ DIAL retrieval using synthetic lidar returns and ozonesondes - Part 1: Algorithm validation
}

\author{
J. T. Sullivan ${ }^{1,4, a}$, T. J. McGee ${ }^{1}$, T. Leblanc ${ }^{2}$, G. K. Sumnicht ${ }^{3}$, and L. W. Twigg ${ }^{3}$ \\ ${ }^{1}$ Atmospheric Chemistry and Dynamics Branch, NASA Goddard Space Flight Center, Greenbelt, MD, USA \\ ${ }^{2}$ California Institute of Technology, Jet Propulsion Laboratory, Wrightwood, CA, USA \\ ${ }^{3}$ Science Systems and Applications Inc., Lanham, MD, USA \\ ${ }^{4}$ Oak Ridge Associated Universities (ORAU), Oak Ridge, TN, USA \\ ${ }^{a}$ formerly at: Department of Atmospheric Physics, University of Maryland, Baltimore County (UMBC) and \\ Joint Center for Earth Systems Technology (JCET), Baltimore, MD, USA \\ Correspondence to: J. T. Sullivan (john.t.sullivan@ nasa.gov)
}

Received: 11 February 2015 - Published in Atmos. Meas. Tech. Discuss.: 28 April 2015

Revised: 28 August 2015 - Accepted: 16 September 2015 - Published: 9 October 2015

\begin{abstract}
The main purpose of the NASA Goddard Space Flight Center TROPospheric OZone DIfferential Absorption Lidar (GSFC TROPOZ DIAL) is to measure the vertical distribution of tropospheric ozone for science investigations. Because of the important health and climate impacts of tropospheric ozone, it is imperative to quantify background photochemical ozone concentrations and ozone layers aloft, especially during air quality episodes. For these reasons, this paper addresses the necessary procedures to validate the TROPOZ retrieval algorithm and confirm that it is properly representing ozone concentrations. This paper is focused on ensuring the TROPOZ algorithm is properly quantifying ozone concentrations, and a following paper will focus on a systematic uncertainty analysis.

This methodology begins by simulating synthetic lidar returns from actual TROPOZ lidar return signals in combination with a known ozone profile. From these synthetic signals, it is possible to explicitly determine retrieval algorithm biases from the known profile. This was then systematically performed to identify any areas that need refinement for a new operational version of the TROPOZ retrieval algorithm. One immediate outcome of this exercise was that a bin registration error in the correction for detector saturation within the original retrieval was discovered and was subsequently corrected for. Another noticeable outcome was that the vertical smoothing in the retrieval algorithm was upgraded from a constant vertical resolution to a variable vertical resolution to yield a statistical uncertainty of $<10 \%$. This new
\end{abstract}

and optimized vertical-resolution scheme retains the ability to resolve fluctuations in the known ozone profile, but it now allows near-field signals to be more appropriately smoothed. With these revisions to the previous TROPOZ retrieval, the optimized TROPOZ retrieval algorithm $\left(\mathrm{TROPOZ}_{\mathrm{opt}}\right.$ ) has been effective in retrieving nearly $200 \mathrm{~m}$ lower to the surface. Also, as compared to the previous version of the retrieval, the $\mathrm{TROPOZ}_{\mathrm{opt}}$ had an overall mean improvement of $3.5 \%$, and large improvements (upwards of $10-15 \%$ as compared to the previous algorithm) were apparent between 4.5 and $9 \mathrm{~km}$. Finally, to ensure the $\mathrm{TROPOZ}_{\mathrm{opt}}$ retrieval algorithm is robust enough to handle actual lidar return signals, a comparison is shown between four nearby ozonesonde measurements. The ozonesondes are mostly within the TROPOZ $\mathrm{opt}_{\text {retrieval un- }}$ certainty bars, which implies that this exercise was quite successful.

\section{Introduction}

It is important to produce validated and quantitative ozone concentration profiles because of the significant climate and health impacts from tropospheric ozone, particularly in the urban environment. Because of this, the ground-based Goddard Space Flight Center TROPospheric OZone DIfferential Absorption Lidar (GSFC TROPOZ DIAL) has been routinely taking measurements in the Baltimore-Washington D.C. region (Greenbelt, $\mathrm{MD} ; 38.99^{\circ} \mathrm{N}, 76.84^{\circ} \mathrm{W} ; 57 \mathrm{~m}$ a.s.l.) 
from a $13 \mathrm{~m}$ transportable trailer since fall of 2013. Many of the instrument specifications and initial TROPOZ retrieval algorithm, which are the baseline retrieval for this paper, can be found in Sullivan et al. (2014). This instrument has been developed as part of the ground-based Tropospheric Ozone Lidar NETwork (TOLNet), which currently consists of five stations across the United States (http://www-air. larc.nasa.gov/missions/TOLNet/). Because this network consists of five different ozone lidar systems, it is important that retrievals for each site be independently validated and compared to provide accurate information for future science campaigns. In May 2014, an intercomparison between the TROPOZ and the Langley Mobile Ozone Lidar (LMOL, Pliutau and De Young, 2013) was performed in which no biases were apparent as compared to ozonesonde profiles when retrievals were performed with adequate signal (Sullivan et al., 2015). Additionally, the TROPOZ and NOAA TOPAZ (Tunable Optical Profiler for Aerosol and oZone lidar, Alvarez et al., 2011) were operated simultaneously for several days in July 2014, and a detailed intercomparison is currently being performed.

The most common method for validation of ozone lidars is sending a balloon-borne electrochemical concentration cell (ECC) instrument through the atmosphere, and it has generally been used as the community validation standard (Thompson et al., 2003). Although launching ECC sondes may be a useful validation tool, the instantaneous ECC measurement may be transported a non-negligible distance away from the ozone lidar resulting in a large difference in air mass. For these reasons, this paper describes the usefulness of utilizing synthetic lidar return signals that are computed using a known ozone profile as an independent validation method in addition to nearby ozonesonde launches. This process also prevents errors in the retrieval process from invalidating quality data. Using simulated lidar data instead of an ozonesonde profile is advantageous because by varying parameters in the modeled return signal, it is possible to explicitly determine both the source and the magnitude of various biases in the retrieval from the original ozone profile (Leblanc et al., 1998; Keckhut et al., 2004a).

This paper addresses the necessary procedures to validate the optimized TROPOZ retrieval algorithm $\left(\mathrm{TROPOZ}_{\mathrm{opt}}\right)$ as compared to the previous Sullivan et al. (2014) algorithm and to confirm that it is properly representing ozone concentrations. A following paper will further focus on a systematic uncertainty analysis with the same proposed methodology. The parameters investigated within this paper are the corrections that occur naturally from spectral properties of trace gases within the atmosphere (including ozone) and limitations of the hardware used to resolve the atmosphere. The numerical derivative is analyzed first to show that it is being performed correctly. Because of naturally varying temperatures in the atmosphere, the temperature dependences of the ozone absorption cross sections are also analyzed. The DIAL measurement involves two wavelengths, and a correction for the differential scattering properties of the Rayleigh atmosphere is also investigated. Additionally, because the detectors reach a regime where they cannot account for the proper amount of return signal, a detector saturation (or pulse pileup) correction is described and analyzed. Vertical resolution is also examined as it can be a controlling factor in representing the correct ozone mixing ratio profile, especially in the upper free troposphere with a decreasing signal to noise ratio (SNR). All of these corrections and refinements were implemented into the new $\mathrm{TROPOZ}_{\mathrm{opt}}$ retrieval algorithm, and the final section of this paper shows a comparison with good agreement with actual ozone retrievals and four nearby ozonesonde profiles.

\section{Synthetic lidar returns and initial retrieval}

In order to validate the GSFC TROPOZ DIAL retrieval algorithm, synthetic lidar return signals have been generated using physical parameters of the lidar system, climatological data, and a known ozone profile. The purpose of generating these synthetic signals is to investigate various parts of the retrieval algorithm with the ability to turn varying effects, such as ambient background radiation, saturation effects, or spectral properties of the atmosphere, on or off. With the ability to vary these effects and decompose the synthetic signals, the TROPOZ retrieval algorithm can be tested in a rigorous manner in order to identify any uncertainty and bias with the original ozone profile.

The synthetic signals were computed using a known atmospheric state produced by the empirical model MSISE90 (Hedin, 1991) between the ground and the thermosphere. The model computes a temperature profile, and profiles of the main atmospheric constituents' number densities including $\mathrm{N}_{2}, \mathrm{O}_{2}$, and Ar for a given day of year and time of day. Additionally, the atmospheric state includes an ozone number density profile computed from a combination of climatologies taken from the UK Universities Global Atmospheric Modelling Programme (UGAMP; Thuburn, 1992) and from the UARS Reference Atmosphere Project.

The simulated lidar return signals are calculated as

$P(i, k)=\frac{P_{L}(i) \eta(i, k) N_{a}(k)}{\left(z(k)-z_{\mathrm{L}}\right)^{2}} \beta(i, k) \tau_{\mathrm{O}_{3}}(i, k) \tau_{M}(i, k)$,

where

$\tau_{\mathrm{O}_{3}}=e^{-\sum_{k^{\prime}=0}^{k} N_{\mathrm{O}_{3}}(k)\left(\sigma_{O_{3} \uparrow}\left(i, k^{\prime}\right)+\sigma_{O_{3} \downarrow}\left(i, k^{\prime}\right)\right) \delta z,}$

$\tau_{M}=e^{-\sum_{k^{\prime}=0}^{k} N_{\mathrm{a}}(k)\left(\sigma_{\mathrm{M} \uparrow}\left(i, k^{\prime}\right)+\sigma_{\mathrm{M} \downarrow}\left(i, k^{\prime}\right)\right) \delta z}$,

and the $i$ and $k$ indices denote the "on" and "off" DIAL wavelength and altitude, respectively. $P$ is the total number of photons collected at each wavelength, $\eta$ is the optical efficiency of the receiving channel, $N_{\mathrm{a}}$ is the air number density, $z$ is the altitude of the backscattering layer, and $z_{\mathrm{L}}$ is the altitude of the lidar system. The backscatter coefficient at each 
layer is denoted as $\beta$, which is currently only from molecular scattering. The optical thickness from the test ozone profile, $\tau_{\mathrm{O}_{3}}$, and from the Rayleigh atmosphere, $\tau_{\mathrm{M}}$, are integrated along the outgoing beam path $(\uparrow)$ and integrated along the returning beam path $(\downarrow)$ between the lidar and the scattering altitude. The test ozone number density profile is $N_{\mathrm{O}_{3}}$, the ozone absorption cross sections are $\sigma_{\mathrm{O}_{3}}$ and the molecular scattering cross sections are $\sigma_{\mathrm{M}}$.

After the atmospheric signals have been generated, the effects of saturation of the detectors and background noise in all channels are computed as

$S(i, k)=\epsilon(i) \frac{P(i, k)}{1+P(i, k)} T_{\mathrm{d}}(i)+P_{\mathrm{b}}(i, k)$,

where $\epsilon$ represents the amplification factor or efficiency of the data recorder system, $T_{\mathrm{d}}$ is the theoretical dead time correction and $P_{\mathrm{b}}$ includes background noise in all channels to account for sky light and electronic noise.

These hardware and atmospheric effects are generated based on various physical components of the TROPOZ lidar system described in Sullivan et al. (2014). Examples of these are the field of view (FOV) of each of the detectors, filter bandwidths, the altitudes at which the signals were gated, and the assumption that the signal can be corrected using a nonparalyzable dead time correction for the photomultiplier tubes (PMTs). The saturation correction is based on the laser repetition rate and the photon counting rate of the data acquisition system. The synthetic signals were also modeled from the standard meteorological atmosphere from the TROPOZ site elevation, latitude, and longitude. In order to properly represent the magnitudes of the synthetic signal, cloud-free, nighttime data are used to simulate realistic return signal levels. Nighttime conditions, where there is naturally a lower level of background noise, allow for a larger vertical range of validation. These acquired signals are then temporally averaged for $10 \mathrm{~min}$ for comparison with the analysis and uncertainty discussion previously reported in Sullivan et al. (2014).

\section{The DIAL equation}

The simulated lidar return signals are not recorded as continuous functions, but rather as values in discrete range bins, $\Delta z$, at the "on" and "off" DIAL wavelengths. It is then possible to write the discrete DIAL equation (Megie et al., 1985) in terms of the range bins specified as

$N_{\mathrm{O}_{3}}(z)=\frac{1}{2 \Delta \sigma_{\mathrm{O}_{3}} \Delta z} \ln \left(\frac{S_{\mathrm{off}}(z+\Delta z)}{S_{\mathrm{off}}(z)} \frac{S_{\mathrm{on}}(z)}{S_{\mathrm{on}}(r+\Delta z)}-C\right)-D$,

where

$\left.C=\frac{\beta_{\mathrm{off}}(z+\Delta z)}{\beta_{\mathrm{off}}(z)} \frac{\beta_{\mathrm{on}}(z)}{\beta_{\mathrm{on}}(z+\Delta z)}\right)$

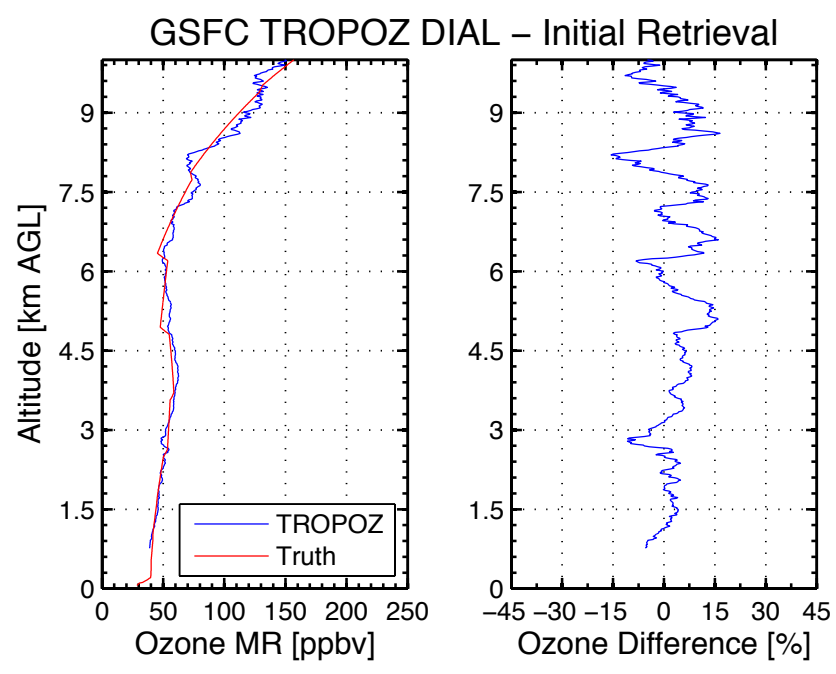

Figure 1. The left panel shows the initial retrieved ozone concentration from the TROPOZ algorithm described in Sullivan et al. (2014) as compared to the known ozone profile used in the simulated lidar return signals. The right panel shows the percent difference from the known profile and retrieved profile.

and with negligible amounts of aerosols and additional interfering gases,

$D=\frac{\Delta \alpha_{M}}{\Delta \sigma_{\mathrm{O}_{3}}}$

For these equations, $\mathrm{N}_{\mathrm{O}_{3}}$ is the ozone number density and $\Delta \sigma_{\mathrm{O}_{3}}$ is the difference in corresponding ozone absorption cross sections taken at the two DIAL wavelengths. The power, atmospheric backscatter coefficient, and atmospheric extinction received from range $z$ at either the "on" or "off" wavelength are denoted as $P, \beta$ and $\alpha$ respectively. The term, $\Delta \alpha_{\mathrm{M}}$, is the difference in the Rayleigh extinction properties of the atmosphere between the two DIAL wavelengths.

The DIAL Eq. (5) is of great interest, and it is of great interest because it lends itself to a self-calibrating technique that can determine the number density of ozone with only the known ozone absorption cross sections and the power returned at each wavelength. The power returned back to the detector is a convolution of backscattered photons from the molecular atmosphere and ambient background sky radiation. Therefore, $P_{\text {off }}$ and $P_{\text {on }}$ are actually comprised of $P_{\text {off }}+P_{\mathrm{b}}$ and $P_{\mathrm{on}}+P_{\mathrm{b}}$, where $P_{\mathrm{b}}$ is the background radiation at the respected DIAL wavelengths.

The correction term $\mathrm{C}$ is due to the spectral difference in the amount of photons that have undergone Rayleigh backscatter into the detectors from the ambient atmosphere. Term D expresses the correction due to the wavelength dependence of Rayleigh extinction and is fairly easily determined with additional meteorological information given by a reference standard atmosphere (US Standard, 1976). With the knowledge of the Rayleigh extinction values, the Rayleigh backscatter term in $\mathrm{C}$ is computed using the as- 
sumed Rayleigh phase function. The implementation of this correction is discussed in a later section of this paper. Although aerosols are not simulated explicitly for this analysis, the aerosol correction discussed in Sullivan et al. (2014) is utilized when comparing the optimized retrieval to actual lidar return signals.

After the TROPOZ retrieval was performed on the synthetic return signals, a final ozone concentration profile was computed. It is then possible to truly compare the final ozone profile to the truth profile originally used to produce the simulated synthetic signals. This is not entirely possible with colocated launches of ozonesondes and shows the advantage of using simulated data as an independent validation source. Investigation of any differences between the TROPOZ retrieval and the true profile can lead to the identification of quantifiable algorithmic biases.

Figure 1 shows the initial TROPOZ retrieval (Sullivan et al., 2014) and its associated ozone differences from the modeled truth profile (red) from $675 \mathrm{~m}$ to $10 \mathrm{~km}$. This is a composite profile which represents two different signal pairs from $675 \mathrm{~m}$ to $2.75 \mathrm{~km}$ and from $2.75 \mathrm{~m}$ to $10 \mathrm{~km}$. The definition of the relative percent difference used for Fig. 1, as well as throughout this paper, is

$\Delta N_{\mathrm{O}_{3}}(\%)=\frac{\operatorname{TROPOZ}_{N_{\mathrm{O}_{3}}}-\operatorname{Model}_{N_{\mathrm{O}_{3}}}}{\operatorname{Model}_{N_{\mathrm{O}_{3}}}} \times 100$.

This retrieval has been performed with a constant $375 \mathrm{~m}$ vertical resolution below $2.75 \mathrm{~km}$ and a $750 \mathrm{~m}$ vertical resolution above $2.75 \mathrm{~km}$. For the region above $4.5 \mathrm{~km}$, this fixed vertical resolution starts to yield large ozone differences near $15 \%$, which can certainly be improved upon and are most likely directly attributed to smoothing effects. Also, near the bottom of the profile and near the join region $(2.75-3 \mathrm{~km})$, there is a comparably large ozone difference which will be discussed in a later section of this work. Although the differences between the initial and final ozone profiles in Fig. 1 are mostly within $15 \%$, there are still underlying biases that may be decreased, and this is the motivation for the following sections of this paper.

\subsection{Numerical derivative}

The first step in ensuring that the DIAL retrieval algorithm is accurate is to confirm that the derivative of the natural logarithm of the ratio of backscattered laser powers from Eq. (5) is correctly calculated. For this reason, a synthetic lidar return signal is simulated to emphasize the use of the numerical derivative. The statistical and background noise, saturation correction, and Rayleigh correction were all removed for this simulation and constant ozone absorption cross sections were used with values of $\sigma_{\mathrm{O}_{3}} 299=4.200 e^{-23} \mathrm{~m}^{2}$ and $\sigma_{O_{3} 289}=1.542 e^{-22} \mathrm{~m}^{2}$ (Malicet et al., 1995).

The finite impulse response (FIR) Savitzky-Golay (SG) differentiation filter (Savitzky and Golay, 1964) used for the

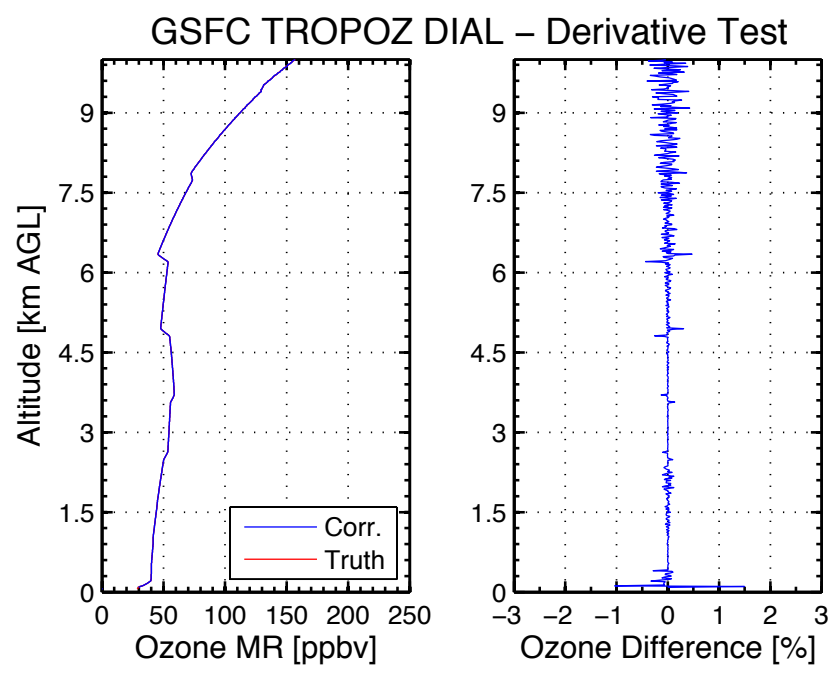

Figure 2. The left panel shows the initial retrieved ozone concentration from the TROPOZ algorithm using the Savitzky-Golay differentiation filter for the numerical derivative as compared to the known ozone profile used in the simulated lidar return signals. The right panel shows the percent difference from the known profile and retrieved profile.

numerical derivative is the same described in Sullivan et al. (2014). The advantage of using the SG filter is that the final vertical resolution of the retrieved ozone can be easily determined using the full width at half maximum (FWHM) of the steady-state SG filter coefficients associated with the filter window size. To emphasize the possible biases from the numerical derivative, the retrieval is done with a minimal three-point smoothing.

The results for using the SG filter are shown in Fig. 2, where the left panel shows the final retrieved ozone mixing ratio from the corrected TROPOZ numerical derivative (blue) as compared to the known ozone profile (red) used in the simulated lidar return signal. Both profiles are in the figure but are directly overtop of each other, implying the numerical derivative is being properly computed in the retrieval algorithm. The right panel shows the negligible percent difference between the known profile and retrieved profile, and this will continue to be used in the new operational version of the TROPOZ $\mathrm{Z}_{\mathrm{opt}}$ ozone retrieval.

\subsection{Temperature dependence of the ozone absorption cross section}

Due to the known temperature dependence of the ozone absorption cross section, it is necessary to get an accurate atmospheric temperature profile, either from a co-located radiosonde launch or from a standard model atmosphere. Ozone absorption cross sections were utilized from Malicet et al. (1995) because of the adequate coverage of standard tropospheric temperatures at the DIAL wavelengths. The $\Delta \sigma_{\mathrm{O}_{3}}$ term is the denominator from Eq. (5), and it is nec- 

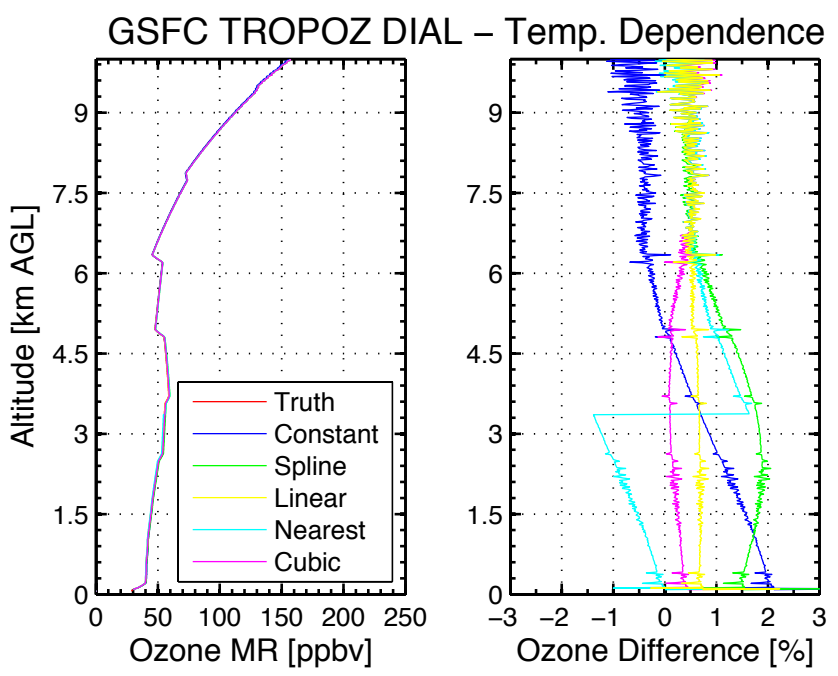

Figure 3. The left panel shows the retrieved ozone mixing ratio from the varying TROPOZ interpolations of the temperature dependence of ozone absorption cross sections as compared to the known ozone profile used in the simulated lidar return signal.The right panel shows the percent difference from the known profile and retrieved profile.

essary to characterize resulting ozone differences from this temperature dependence. Because the ozone absorption temperature dependence is not known continuously but rather at discrete temperatures, various interpolations have been investigated and are all shown.

In the left panel of Fig. 3, ozone mixing ratios are retrieved using the constant ozone absorption cross sections of $\sigma_{O_{3} 299}=4.200 e^{-23} \mathrm{~m}^{2}$ and $\sigma_{O_{3} 289}=1.542 e^{-22} \mathrm{~m}^{2}$, but with varying temperature interpolations (Malicet et al., 1995). The statistical and background noise, saturation correction and Rayleigh correction were all removed for this simulation. One profile corresponds to constant values of $\Delta \sigma_{\mathrm{O}_{3}}$, and additional profiles use a different interpolation of the ozone absorption cross sections. Although the final mixing ratios look very similar for each temperature interpolation, the right panel of Fig. 3 shows subtle differences between the various interpolation schemes (Boor, 1978). For spline fitting, the interpolated value at a query point is based on a cubic interpolation of the values using not-a-knot conditions at neighboring grid points. For linear and cubic fitting, the interpolated value at a query point is based on linear and cubic interpolation of the values. For nearest fitting, the interpolated value at a query point is the value at the nearest sample grid point.

Regardless of the interpolation used for the synthetic return, the final ozone differences are all mostly within $2 \%$ of the known ozone profile. The blue line, representing a constant temperature value, emphasizes the importance of correcting the TROPOZ retrieval algorithm for temperature, especially in the first few kilometers of the troposphere. The

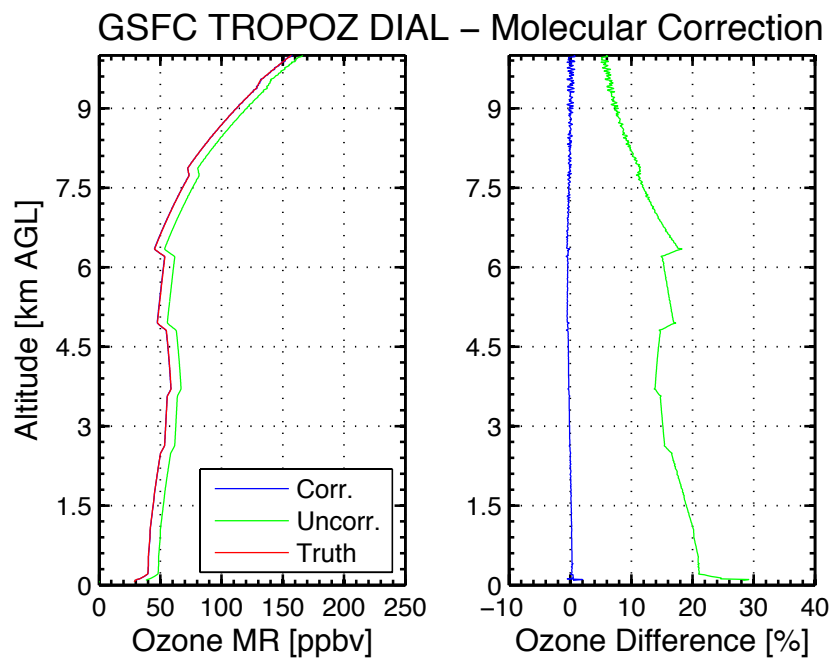

Figure 4. The left panel shows the retrieved ozone mixing ratios from the corrected and uncorrected TROPOZ ozone profiles as compared to the known ozone profile used in the simulated lidar return signal.The right panel shows the percent difference from the known profile and retrieved profile.

lower portion of this region, known as the planetary boundary layer (PBL), has many stratified temperature layers and inversions, in which an accurate ozone mixing ratio requires an interpolated scheme. Based on the right panel in Fig. 3, most of these interpolations yield a similar bias (within $\pm 1.0 \%$ ) throughout the lower free troposphere.

Although these percentage differences are based on the difference between the cross sections used in the synthetic simulation and the retrieval algorithm, it is important to quantify the magnitude of the bias associated with using a constant cross section and with each of the various interpolations. Based on the biases shown from these interpolations, the $\mathrm{TROPOZ}_{\mathrm{opt}}$ retrieval algorithm will implement the cubic interpolation of the temperature dependence of the ozone absorption cross sections. Aside from computing ozone profiles with Malicet et al. (1995), there are other sources for the ozone absorption cross sections, which have been discussed throughout WMO (2015). The differences within the tropospheric temperature range between the data sets in this report are mostly within $5 \%$ of each other at the DIAL wavelengths used in this study.

\subsection{Rayleigh molecular extinction}

After the analysis of the previous parameter changes, the DIAL Eq. (5) was satisfactorily investigated. The corrections for Rayleigh molecular backscatter (Eq. 6) and extinction (Eq. 7), which are based on spectral properties of the atmosphere, are now implemented in the simulated data. The statistical and background noise were removed for this simulation. The saturation corrections were also removed, and constant ozone absorption cross sections were 

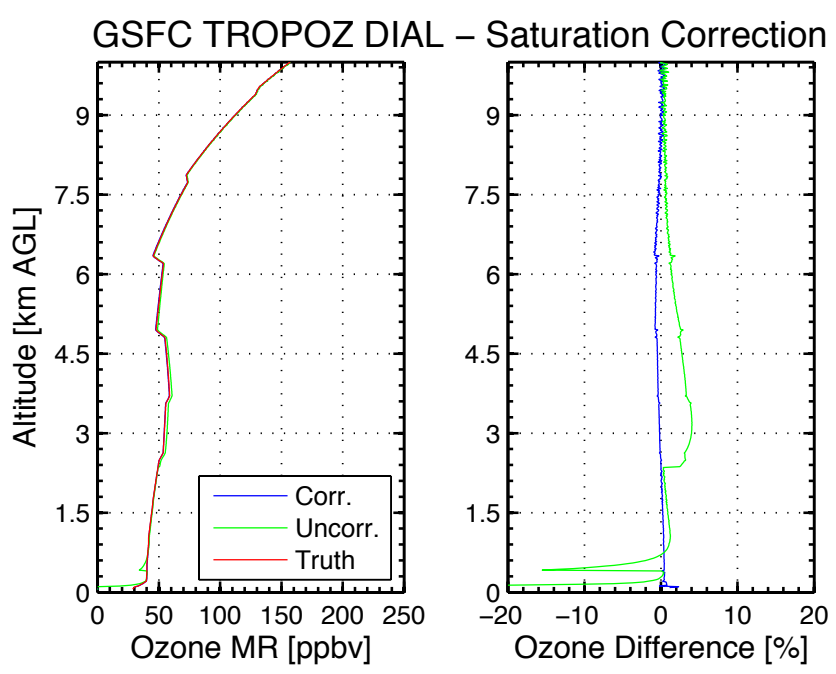

Figure 5. The left panel shows the retrieved ozone mixing ratios from the saturation corrected and uncorrected TROPOZ ozone profiles as compared to the known ozone profile used in the simulated lidar return signal.The right panel shows the percent difference from the known profile and retrieved profile.

used with values of $\sigma_{O_{3} 299}=4.200 e^{-23} \mathrm{~m}^{2}$ and $\sigma_{O_{3} 289}=$ $1.542 e^{-22} \mathrm{~m}^{2}$. The correction from Eq. (7) is calculated with the simulated atmospheric number density and constant values of Rayleigh extinction cross sections of $\alpha_{\text {mol2 } 299}=$ $5.730 e^{-30} \mathrm{~m}^{2}$ and $\alpha_{\mathrm{mol} 289}=6.661 e^{-30} \mathrm{~m}^{2}$ (Eberhard, 2010). The Rayleigh backscatter volume cross sections in Eq. (6) are then computed from the Rayleigh phase function, $\alpha_{\text {mol2 } 29}$ and $\alpha_{\text {mol289. }}$.

The left panel of Fig. 4 shows the corrected, uncorrected and known ozone mixing ratio profile. The right panel of Fig. 4 shows the percent difference for the corrected and uncorrected profiles. Once again, similar to Fig. 2, the corrected and truth profiles are almost identical. Without this correction, the magnitude of this correction is near $20 \%$ in the PBL and $10 \%$ in the free troposphere. This is much more substantial than the temperature dependence of the ozone absorption cross sections, but the correction only varies largely with atmospheric number density and is therefore typically easy to correct for. The ozone difference plot in the right panel shows that this correction is $<1 \%$ if the atmospheric number density is precisely known. For this reason the $\mathrm{TROPOZ}_{\mathrm{opt}}$ retrieval will implement the updated Rayleigh extinction cross sections.

\subsection{Saturation (pulse pile-up)}

The TROPOZ retrieval algorithm must also correct for the nonparalyzable dead time correction of the PMTs (Keckhut et al., 2004b). The values used in this simulation are based on the theoretical maximum photon counting rate of the data

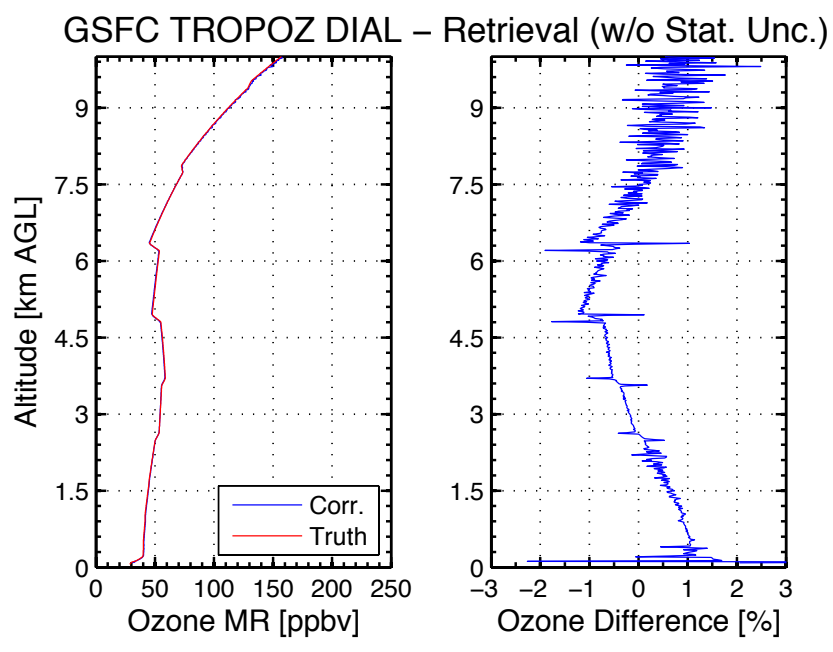

Figure 6. The left panel shows the retrieved ozone mixing ratios from the $\mathrm{TROPOZ}_{\mathrm{opt}}$ ozone profiles as compared to the known ozone profile used in the simulated lidar return signal without the addition of statistical noise. The right panel shows the percent difference from the known profile and retrieved profile.

acquisition system, which is $250 \mathrm{MHz}$ or $4.0 \mathrm{~ns}$. This correction can be applied as

$C_{\mathrm{t}}=\frac{C_{\mathrm{m}}}{1-C_{m} T_{\mathrm{d}}}$,

where the true photon count rate $\left(C_{\mathrm{t}}\right)$ can be expressed as a function of the measured count rates $\left(C_{\mathrm{M}}\right)$ and a dead time $\left(T_{\mathrm{d}}\right)$ parameter (Lampton and Bixler, 1985).

When this theoretical value was used with the current retrieval, it did not appear to completely correct for the detector saturation (pulse pile-up). Upon looking at this closer, a bin registration issue was found in the algorithm and was subsequently adjusted to correctly implement Eq. (9).

The left panel of Fig. 5 shows the corrected, uncorrected and truth profiles for the known ozone profile. The right panel shows the percent difference between each of these profiles. The saturation correction is particularly important in the lower regions of each channel, and an improper algorithm may lead to biases upwards of $20 \%$. Based on the percent difference plot in the right side of Fig. 5, the difference in this correction is $<1 \%$ and this will be implemented in the $\mathrm{TROPOZ}_{\mathrm{opt}}$ ozone retrieval.

\subsection{TROPOZ $_{\text {opt }}$ retrieval algorithm before the addition of statistical noise}

After carrying out each of these corrections, it was important to compare the TROPOZ truth profile used in the simulation. The left panel of Fig. 6 shows the new corrected $\mathrm{TROPOZ}_{\mathrm{opt}}$ retrieval and truth profiles for the known ozone concentration profile. The right panel shows the percent difference between the $\mathrm{TROPOZ}_{\mathrm{opt}}$ 
retrieval and known profile without the addition of statistical noise. The spikes in the right panel correspond to abrupt ozone gradients in the simulated ozone profile and are not expected to occur as sharply in the natural atmosphere.

Although it would be physically impossible to determine this percent difference in the real atmosphere with an ozonesonde, this exercise allows the $\mathrm{TROPOZ}_{\mathrm{opt}}$ retrieval to biases to be completely quantified before real atmospheric noise is involved. The percent differences have been quantified to be mostly within $\pm 1 \%$ of the known ozone profile. With the addition of statistical noise, these biases can grow much larger and an optimization scheme is shown in the following section.

\section{TROPOZ $_{\text {opt }}$ variable vertical-resolution scheme and uncertainty analysis}

As mentioned before, the TROPOZ retrieval algorithm originally implemented a constant vertical resolution of $375 \mathrm{~m}$ below $2.75 \mathrm{~km}$ and $750 \mathrm{~m}$ above $2.75 \mathrm{~km}$. The left panel of Fig. 7 depicts the new TROPOZ ${ }_{\text {opt }}$ retrieval effective vertical-resolution scheme. These values are coupled directly to the FWHM of the steady-state SG filter coefficients associated with the window size as described in the "Numerical derivative" section of this paper.

Because a bias naturally occurs due to the decrease in the SNR with altitude, it is favorable to increase the number of points of the derivative low-pass filter used for data processing (Godin et al., 1999). This is evident in the large difference near the $3 \mathrm{~km}$ join region, in which the lower channel's SNR is decreasing and more data points are needed to provide an accurate ozone profile. However, the adjoining upper channel has a sufficiently high SNR to properly perform the retrieval. The large gradient in the SNR, and therefore the vertical resolution, can mostly be attributed to physical hardware parameters in the TROPOZ system such as transmitted laser pulse power, telescope diameter, FOV, and various optical filters. Although Fig. 7 presents an optimized verticalresolution scheme for the hardware of the lidar system, it also allows higher-resolution data throughout the dynamic PBL in order to characterize ozone features.

The TROPOZ detects individual photons through the use of photon counting and PMTs. The signal collected by these PMTs follows Poisson statistics (Megie et al., 1985; Papayannis et al., 1990), and the statistical uncertainty of the ozone concentrations is shown in the right panel of Fig. 7. The statistical uncertainty at a given range can be calculated as

$\epsilon_{N_{\mathrm{O}_{3}}}(i, z)=\frac{1}{2 N_{\mathrm{O}_{3}} \Delta \sigma_{\mathrm{O}_{3}} \Delta z_{e}} \sqrt{\frac{S(i, z)+P_{\mathrm{b}}(i, z)+P_{\mathrm{d}}(i, z)}{S(i, z)^{2}}}$,

where $S, P_{\mathrm{b}}$, and $P_{\mathrm{d}}$ are the atmospheric backscattered signal, background radiation, and dark counts of the detector
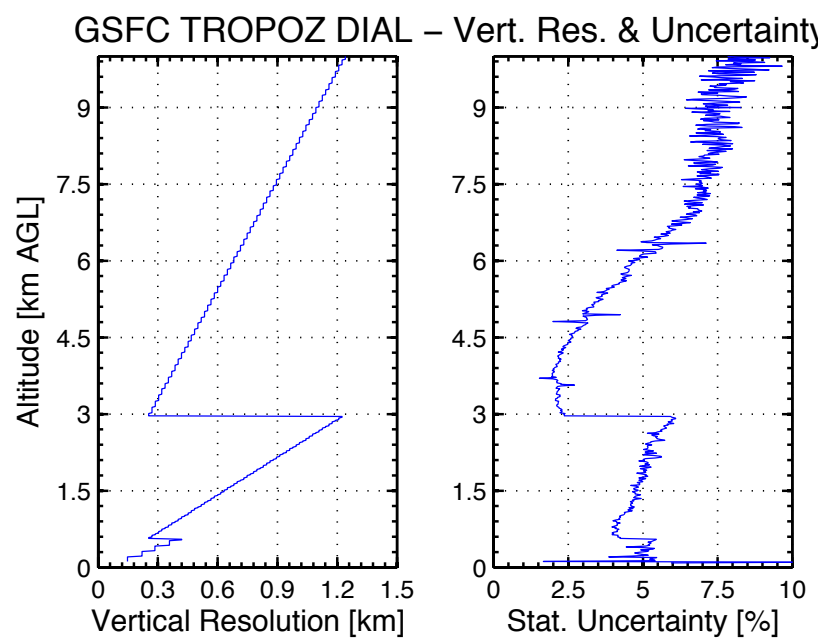

Figure 7. The left panel shows the associated vertical resolution in the $\mathrm{TROPOZ}_{\mathrm{opt}}$ retrieval algorithm, which is derived from the window size of the SG differentiation filter. The right panel shows the statistical uncertainty in the system associated with these vertical resolutions, which is an approximation for the overall uncertainty for the $\mathrm{TROPOZ}_{\mathrm{opt}}$.

at the DIAL wavelength $i$. The total statistical uncertainty is then calculated from the statistical uncertainty from each DIAL wavelength added in quadrature. The effective vertical resolution, which is based on the SG filter window size, is denoted as $\Delta z_{e}$ and the differential ozone absorption cross section is denoted as $\Delta \sigma_{\mathrm{O}_{3}}$.

The statistical uncertainty is related to the square root of the total PMT counts, both those that are relevant to the retrieval of ozone number density and those that are counts due to systematic uncertainties. Although this analysis was performed with a $10 \mathrm{~min}$ average of simulated data, by integrating profiles for a longer duration, the backscattered signal term $S$ becomes much larger than the $P_{\mathrm{b}}$ and $P_{\mathrm{d}}$ terms. For this reason, the temporal resolution is inherently built into the statistical uncertainty of the system and averaging many data sets is beneficial to the resultant uncertainty in the system.

The right panel of Fig. 7 also shows that by increasing the smoothing window used in the retrieved ozone profile, which is in the denominator of Eq. (10), the statistical uncertainty in the measurement can be maintained within a desired limit. Because the vertical resolution changes with altitude and has different values for different channel pairs, the resultant uncertainty profile exhibits the analogous changes. This allows for an optimized vertical-resolution scheme to obtain a final statistical uncertainty in the system that is $<10 \%$. Although a more rigorous and detailed uncertainty analysis will be discussed in the next installment of this paper, the right panel of Fig. 7 shows an approximation for the overall uncertainty for

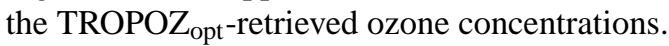




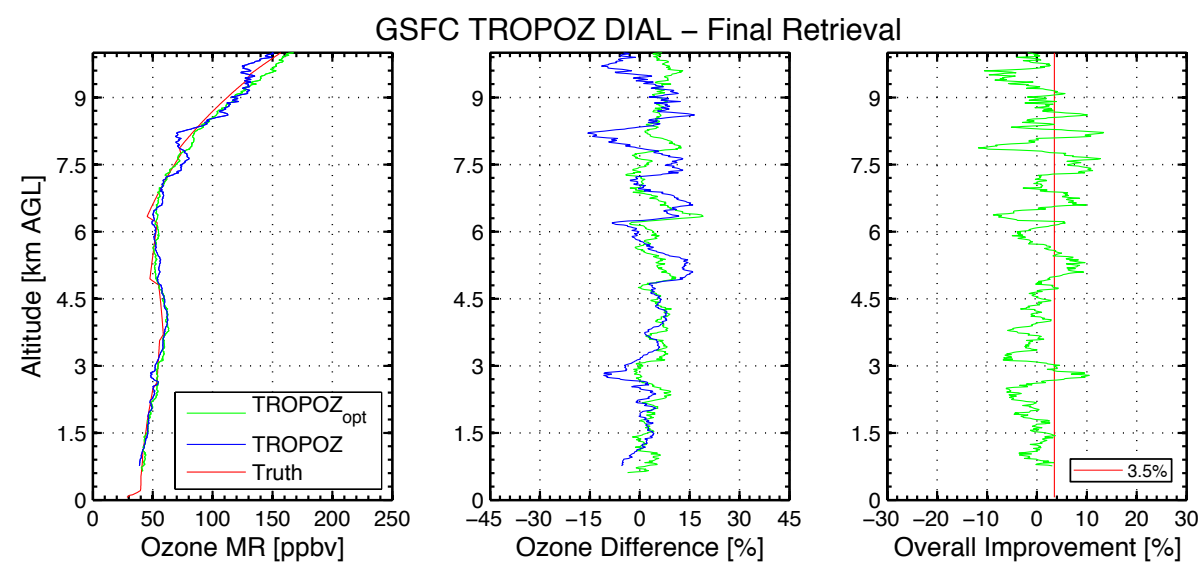

Figure 8. The left panel compares the final retrieved ozone concentration from the previous TROPOZ retrieval and the optimized retrieval $\left(\mathrm{TROPOZ}_{\mathrm{opt}}\right.$ ) to the known ozone profile used in the simulated lidar return signal. The center panel shows the differences in percentage from the known profile and retrieved $\mathrm{TROPOZ}$ and $\mathrm{TROPOZ}_{\mathrm{Opt}}$ profiles. The right panel shows the improvement from the optimized $\mathrm{TROPOZ}_{\mathrm{opt}}$ retrieval and the original TROPOZ retrieval in percentage. The mean improvement (red line) is $3.5 \%$.

Table 1. Summary of the improvements associated with the optimized GSFC TROPOZ DIAL algorithm as compared to the initial algorithm in Sullivan et al. (2014).

\begin{tabular}{lrrr}
\hline & $\begin{array}{r}\text { Low channel } \\
(0-0.5 \mathrm{~km})\end{array}$ & $\begin{array}{r}\text { Middle channel } \\
(0.5-2.75 \mathrm{~km})\end{array}$ & $\begin{array}{r}\text { High channel } \\
(>2.75 \mathrm{~km})\end{array}$ \\
\hline Absorption cross-section interpolation & $1-2 \%$ & $1-2 \%$ & $<1 \%$ \\
Rayleigh correction & $<1 \%$ & $<1 \%$ & $<1 \%$ \\
Saturation correction & $5-10 \%$ & $1-5 \%$ & $1-5 \%$ \\
Statistical uncertainty $^{\mathrm{a}}$ & $1-5 \%$ & $1-11 \%$ & $1-15 \%$ \\
\hline
\end{tabular}

${ }^{a}$ Improvements due to optimization of the vertical resolution (Fig. 7).

\section{Comparison of the original TROPOZ and $\mathrm{TROPOZ}_{\text {opt }}$ retrieval algorithms}

The left panel of Fig. 8 shows the original TROPOZ retrieval (Fig. 1), the optimized TROPOZ retrieval $\left(\mathrm{TROPOZ}_{\mathrm{opt}}\right)$ and the truth ozone profile. The $\mathrm{TROPOZ}_{\mathrm{opt}}$ retrieval has implemented all of the changes and corrections described throughout the previous sections of this paper including the optimized vertical-resolution scheme from Fig. 7.

The middle panel of Fig. 8 shows the percent difference between each of the retrievals and the truth profile. Due to the optimized vertical smoothing scheme, the TROPOZ $\mathrm{Opt}_{\text {algo- }}$ rithm is able to produce ozone profiles nearly $200 \mathrm{~m}$ lower (from 675 to $500 \mathrm{~m}$ ) than the previous TROPOZ retrieval. The bin registration error that was identified with the saturation correction is also adjusted in the final $\mathrm{TROPOZ}_{\mathrm{opt}} \mathrm{re}-$ trieval. This adjustment shows a direct reduction in percent difference near the retrieval join regions of nearly $5 \%$ from 675 to $800 \mathrm{~m}$ and $10 \%$ from 2.75 to $3 \mathrm{~km}$. This panel also shows reductions mostly between 5 and $15 \%$ in the percent difference of the upper tropospheric retrieval as compared to the previous algorithm.
The right panel in Fig. 8 serves as a visual summary to quantify the improvement gained from this optimization process. The improvement was calculated from the difference in the absolute value of each difference profile in the middle panel Fig. 8 and can be written as

$$
\text { Improvement }_{\%}=|\mathrm{TROPOZ} \%|-\left|\mathrm{TROPOZ} \mathrm{opt}_{\%}\right| .
$$

The overall profile mean improvement from the original retrieval to the $\mathrm{TROPOZ}_{\mathrm{opt}}$ retrieval (red line) is $3.5 \%$. In terms of ozone concentrations, this mean improvement is somewhere between 2 and 4 ppbv. The largest improvements occur in the upper atmosphere where the retrieval performance and vertical resolution were optimized. Specifically, some of the retrieved ozone concentrations above $4.5 \mathrm{~km}$ have improved greatly by more than $10 \%$.

Table 1 indicates the improvement of the optimized algorithm as compared to the initial TROPOZ algorithm described in Sullivan et al. (2014). There is a 1-2\% improvement with the updated interpolation scheme for the ozone absorption cross section. Improvements in the uncertainty related to the saturation effects at all altitudes have been improved, largely because of the application of the proper cor- 

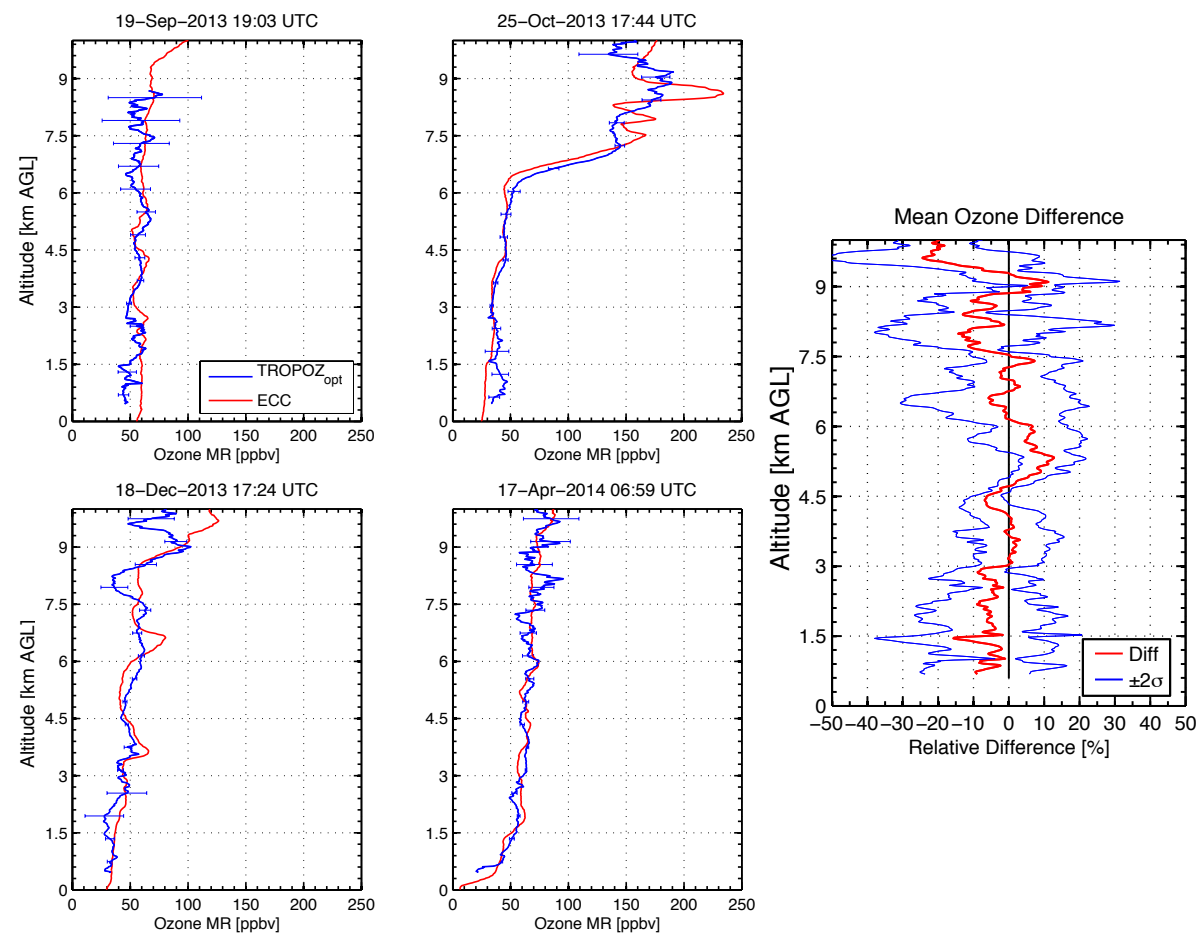

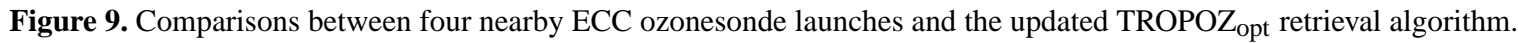

rection discovered during this process. The improvement is largest, $5-10 \%$, in the low channel because the signals are nearest to saturation, but still had a non-negligible effect, 1$5 \%$, in the middle and high channels. As discussed previously, large improvements, upwards of $10-15 \%$, in the middle and high channels corresponded directly to the optimized vertical-resolution scheme.

\section{Final TROPOZ $\mathrm{Z}_{\text {opt }}$ retrieval as compared to ozonesondes}

After implementing the $\mathrm{TROPOZ}_{\mathrm{opt}}$ retrieval algorithm it was important to analyze real lidar signals profiles that are convolved with sources of noise. This allows for confirmation that the real ambient sky radiation is being correctly accounted for in the final ozone mixing ratio profile. The aerosol correction from Sullivan et al. (2014) has also been applied to the real lidar signals. Although the theoretical dead time correction value is $4.00 \mathrm{~ns}$, based on the counting rate of the transient recorder, this is rarely physically achieved. For this reason, larger values between 4 and $5 \mathrm{~ns}$ are used and were empirically determined by comparing the lidar return signal to a model atmosphere or from ozonesonde data.

Figure 9 shows four different ozonesonde launches as compared to the new $\mathrm{TROPOZ}_{\mathrm{opt}}$ algorithm, and the uncertainty bars represent the statistical uncertainty of the measurement described in Fig. 7. These lidar profiles are $10 \mathrm{~min}$ averages and are each centered around 19 September 2013 at 19:03 UTC, 25 October 2013 at 17:44 UTC, 18 December 2013 at 17:24 UTC, or 17 April 2014 at 06:59 UTC. The ozonesondes were launched by the Howard University Beltsville Center for Climate Systems Observation. The launch site $\left(39.05^{\circ} \mathrm{N}, 76.88^{\circ} \mathrm{W}\right)$ is approximately $8 \mathrm{~km}$ from the lidar site which is close enough to assume similar but not identical tropospheric micrometeorology in the dynamic daytime PBL. These comparison times were chosen to maximize overlap of the two instruments based on the sonde's proximity to the lidar and ascent rate.

In each of the cases, the $\mathrm{TROPOZ}_{\mathrm{opt}}$ retrieval was able to produce good agreement with the instantaneous ozonesonde profile from $300 \mathrm{~m}$ to $10 \mathrm{~km}$. The first $\mathrm{TROPOZ}_{\mathrm{opt}}$ retrieval on 19 September 2013 at 19:03 UTC shows the largest uncertainty and deviation from the ozonesonde profile than in any of the other four profiles. This is largely because it was retrieved before a hardware modification was made, in which an additional detector was added to better resolve the upper atmosphere. This is an example of how the statistical uncertainty can grow rapidly as the SNR of the system decreases. The following profile on 25 October 2013 at 17:44 UTC shows good agreement between the ozonesonde and the $\mathrm{TROPOZ}_{\mathrm{opt}}$ retrieval, with the largest uncertainty occurring near $10 \mathrm{~km}$. At this altitude, ozone mixing ratio values reaching near $200 \mathrm{ppbv}$ were resolved. This large gradient would not have been resolved as accurately with the original vertical-resolution scheme. On 18 December 2013 at 17:24 UTC the TROPOZ $_{\text {opt }}$ retrieval shows good agreement 
with the ozonesonde profile for the lower altitude ranges, but it begins to differ in the upper altitudes. The final ozonesonde comparison on 17 April 2014 at 06:59 UTC shows excellent agreement between the ozonesonde and the $\mathrm{TROPOZ}_{\mathrm{Opt}^{-}}$ retrieved ozone mixing ratio. This a nighttime ozonesonde launch, in which the sky background radiation is negligible and the SNR is naturally higher. These combine with a very low concentration of ozone to yield a fairly low statistical uncertainty in the measurement.

\section{Conclusions}

This paper serves as the first paper to concentrate on the optimization of the GSFC TROPOZ DIAL retrieval. This paper is focused on ensuring that the TROPOZ algorithm is accurately quantifying ozone concentrations, and the following paper will focus on a robust uncertainty analysis. Using simulated lidar returns has shown to be beneficial for testing a new operational version of TROPOZ analysis algorithm. The advantage of using simulated signals is that it is possible to turn varying effects on and off in order to investigate differences between the retrieval and the known truth profile. These differences could never have been truly investigated with actual lidar returns and instantaneous ozonesonde profiles because the state of the atmosphere is never precisely known.

One key improvement from this analysis came from optimizing the vertical-resolution scheme from a previously constant resolution. These improvements were upwards of $10 \%$ above $4.5 \mathrm{~km}$. The overall improvement was $3.5 \%$ from the previous retrieval, and it was able to extend the lower limit of the range of ozone retrievals by nearly $200 \mathrm{~m}$. The authors believe that this analysis has significantly added to the confidence that the TROPOZ retrieval algorithm is properly quantifying ozone concentrations. Application of this technique will be recommended to all other TOLNet lidars for validation, optimization, and consistency purposes.

Acknowledgements. This work was supported by UMBC/JCET (Task \#374, Project 8306), the Maryland Department of the Environment (MDE, Contract \#U00P4400079), NOAA-CREST CCNY Foundation (Sub-Contract \#49173B-02) and the National Aeronautics and Space Administration. Work at the Jet Propulsion Laboratory, California Institute of Technology, was carried out under contract with the National Aeronautics and Space Administration. The authors gratefully acknowledge support provided by NASA HQ, the NASA Tropospheric Chemistry Program and the Tropospheric Ozone Lidar Network (TOLNet). Thanks to Raymond M. Hoff for additional support and knowledge of lidar techniques. Also, thanks to the Howard University - Beltsville Center for Climate Systems Observation for launching the ozonesondes necessary to continue validating this system.

Edited by: G. Pappalardo

\section{References}

Alvarez, R. J., Senff, C. J., Langford, A. O., Weickmann, A. M., Law, D. C., Machol, J. L., Merritt, D. A., Marchbanks, R. D., Sandberg, S. P., Brewer, W. A., Hardesty, R. M., and Banta, R. M.: Development and application of a compact, tunable, solidstate airborne ozone lidar system for boundary layer profiling, J. Atmos. Ocean.Technol., 28, 1258-1272, 2011.

Boor, C. D.: A practical guide to splines, Mathematics of Computation, 1978.

Eberhard, W. L.: Correct equations and common approximations for calculating Rayleigh scatter in pure gases and mixtures and evaluation of differences, Appl. Opt., 49, 1116-1130, 2010.

Godin, S., Carswell, A. I., Donovan, D. P., Claude, H., Steinbrecht, W., McDermid, I. S., McGee, T. J., Gross, M. R., Nakane, H., Swart, D. P. J., Bergwerff, H. B., Uchino, O., von der Gathen, P., and Neuber, R.: Ozone differential absorption lidar algorithm intercomparison, Appl. Opt., 38, 6225-6236, 1999.

Hedin, A. E.: Extension of the MSIS thermosphere model into the middle and lower atmosphere, J. Geophys. Res.-Space, 96, 1159-1172, 1991.

Keckhut, P., McDermid, S., Swart, D., McGee, T., GodinBeekmann, S., Adriani, A., Barnes, J., Baray, J.-L., Bencherif, H., Claude, H., di Sarra, A. G., Fiocco, G., Hansen, G., Hauchecorne, A., Leblanc, T., Lee, C. H., Pal, S., Megie, G., Nakane, H., Neuber, R., Steinbrecht, W., and Thayer, J.: Review of ozone and temperature lidar validations performed within the framework of the Network for the Detection of Stratospheric Change, J. Environ. Monit., 6, 721-733, doi:10.1039/B404256E, 2004a.

Keckhut, P., McDermid, S., Swart, D., McGee, T., GodinBeekmann, S., Adriani, A., Barnes, J., Baray, J.-L., Bencherif, H., Claude, H., di Sarra, A. G., Fiocco, G., Hansen, G., Hauchecorne, A., Leblanc, T., Lee, C. H., Pal, S., Megie, G., Nakane, H., Neuber, R., Steinbrecht, W., and Thayer, J.: Review of ozone and temperature lidar validations performed within the framework of the Network for the Detection of Stratospheric Change, J. Environ. Monit., 6, 721-733, doi:10.1039/B404256E, $2004 b$.

Lampton, M. and Bixler, J.: Counting efficiency of systems having both paralyzable and nonparalyzable elements, Rev. Sci. Instrum., 56, 164-165, 1985.

Leblanc, T., McDermid, I. S., Hauchecorne, A., and Keckhut, P.: Evaluation of optimization of lidar temperature analysis algorithms using simulated data, J. Geophys. Res.-Atmos., 103, 6177-6187, doi:10.1029/97JD03494, 1998.

Malicet, J., Daumont, D., Charbonnier, J., Parisse, C., Chakir, A., and Brion, J.: Ozone UV spectroscopy. II. Absorption crosssections and temperature dependence, J. Atmos. Chem., 21, 263 273, doi:10.1007/BF00696758, 1995.

Megie, G. J., Ancellet, G., and Pelon, J.: Lidar measurements of ozone vertical profiles, Appl. Opt., 24, 3454-3463, 1985.

Papayannis, A., Ancellet, G., Pelon, J., and Mégie, G.: Multiwavelength lidar for ozone measurements in the troposphere and the lower stratosphere, Appl. Opt., 29, 467-476, 1990.

Pliutau, D. and De Young, R.: UV lidar receiver analysis for tropospheric sensing of ozone, Technical Manual 218038, NASA, 2013. 
Savitzky, A. and Golay, M. J. E.: Smoothing and differentiation of data by simplified least squares procedures, Anal. Chem., 36, 1627-1639, 1964.

Sullivan, J. T., McGee, T. J., Sumnicht, G. K., Twigg, L. W., and Hoff, R. M.: A mobile differential absorption lidar to measure sub-hourly fluctuation of tropospheric ozone profiles in the Baltimore-Washington, D.C. region, Atmos. Meas. Tech., 7, 3529-3548, doi:10.5194/amt-7-3529-2014, 2014.

Sullivan, J. T., McGee, T. J., De Young, R., Sumnicht, G. K., Twigg, L. W., Pliutau, D., Carrion, W., and Knepp, T.: Results from the NASA GSFC and LaRC ozone lidar intercomparison: New mobile tools for atmospheric research, J. Atmos. Ocean. Technol., 32, 1779-1795, 2015.

Thompson, A. M., Witte, J. C., McPeters, R. D., Oltmans, S. J., Schmidlin, F. J., Logan, J. A., Fujiwara, M., Kirchhoff, V. W. J. H., Posny, F., Coetzee, G. J. R., Hoegger, B., Kawakami, S., Ogawa, T., Johnson, B. J., Vömel, H., and Labow, G.: Southern Hemisphere Additional Ozonesondes (SHADOZ) 1998-2000 tropical ozone climatology 1. Comparison with Total Ozone Mapping Spectrometer (TOMS) and ground-based measurements, J. Geophys. Res.-Atmos., 108, D2, doi:10.1029/2001JD000967, 2003.
Thuburn, J.: UGAMP Internal Report, Tech. Rep. 16, University of Exeter, UK, 1992.

US Standard: US standard atmosphere, 1976, Adopted by the United States Committee on Extension to the Standard Atmosphere, National Oceanic and Amospheric Administration, US Govt. Print. Off., Washington, 1976.

WMO: Absorption Cross-Sections of Ozone (ACSO), Global Atmospheric Watch Report No. 218, Tech. rep., World Meteorological Institution, 2015. 